\title{
The relationship between total plasma carotenoids and risk factors for chronic disease among middle-aged and older men
}

\author{
Wildon R. Farwell ${ }^{1,2} *$, J. Michael Gaziano ${ }^{1,2,3}$, Edward P. Norkus ${ }^{4}$ and Howard D. Sesso ${ }^{2,3}$ \\ ${ }^{1}$ Massachusetts Veterans Epidemiology Research and Information Center, VA Boston Healthcare System, Boston Division \\ (151 MAV), 150 South Huntington Avenue, Boston, MA, USA \\ ${ }^{2}$ Division of Aging, Department of Medicine, Brigham and Women's Hospital and Harvard Medical School, Boston, MA, USA \\ ${ }^{3}$ Division of Preventive Medicine, Department of Medicine, Brigham and Women's Hospital, Boston, MA, USA \\ ${ }^{4}$ Departments of Medical Research, Our Lady of Mercy Medical Center and Community and Preventive Medicine, New York \\ Medical College, Bronx, NY, USA
}

(Received 12 June 2007 - Revised 19 November 2007 - Accepted 14 January 2008 - First published online 12 March 2008)

Individual plasma carotenoids have been associated with various chronic diseases but little is known about the relationship between total plasma carotenoids and risk factors for chronic diseases. In the Physicians' Health Study, we examined 492 men free of CVD and cancer for the relationship between total plasma carotenoids (the sum of $\alpha$-carotene, $\beta$-carotene, lycopene, zeaxanthin, lutein and $\beta$-cryptoxanthin) and a wide variety of factors that predict chronic disease. Multivariate linear and logistic regression was performed to calculate parameter estimates $(95 \% \mathrm{CI})$ and OR $(95 \% \mathrm{CI})$ for total plasma carotenoids. In linear regression models, BMI, hypertension, alcohol intake and plasma levels of each lipid parameter and $\alpha$-tocopherol significantly predicted levels of total plasma carotenoids. Upon adjustment for multiple chronic disease risk factors, the OR for levels of total plasma carotenoids greater than or equal to the median $(\geq 1.301 \mu \mathrm{mol} / \mathrm{l})$ was statistically significant for current smoking (OR 0.21; $95 \%$ CI 0.06, 0.77), weekly alcohol ingestion (OR 2.30; $95 \%$ CI 1.06, 4.99), daily alcohol ingestion (OR 2.46; $95 \%$ CI 1.29, 4.67), each $100 \mathrm{mg} / \mathrm{l}$ increase in total cholesterol (OR 0.73; $95 \%$ CI 0.58, 0.91), LDL-cholesterol (OR 1.48; 95\% CI 1.17, 1.89) and HDL-cholesterol (OR 1.58; $95 \%$ CI 1.26, 1.99), each $100 \mathrm{mg} / \mathrm{ml}$ increase in intercellular adhesion molecule-1 (OR 0.70 ; $95 \%$ CI 0.53 , 0.93 ) and each $10 \mu \mathrm{mol} / \mathrm{l}$ increase in $\alpha$-tocopherol (OR 1.33; $95 \%$ CI 1.12, 1.57), using logistic regression. Few lifestyle and clinical risk factors appear to be related to levels of total plasma carotenoids; however, levels of biomarkers such as plasma lipids and $\alpha$-tocopherol may be strongly related.

Carotenoids: Risk factors: Chronic disease

Higher levels of fruit and vegetable intake have been associated with a decreased risk of various chronic medical conditions $^{(1)}$. The antioxidant properties of carotenoids, commonly found in fruits and vegetables, are one explanation for this finding. Increased dietary carotenoid consumption and levels of individual plasma carotenoids may be associated with the decreased risk of cancer and $\mathrm{CVD}^{(2,3)}$. In addition, levels of individual plasma carotenoids have been associated with specific risk factors for chronic medical conditions such as diet, exercise and cholesterol ${ }^{(4-7)}$. Some authors have suggested using total plasma carotenoids as a marker of a diet high in fruits and vegetables ${ }^{(8)}$. However, there are comparatively fewer data regarding total plasma carotenoids and the frequency of both traditional and more novel risk factors for chronic medical conditions. Therefore, we examined the association between various traditional and more novel risk factors with total plasma carotenoids in a cross-sectional sample of middle-aged and older men.

\begin{abstract}
Methods
Study population

The Physicians' Health Study (PHS) has previously been described in detail ${ }^{(9)}$. Briefly, the PHS began in 1982 as a randomised, double-blind, placebo-controlled, $2 \times 2$ factorial trial of $\beta$-carotene and aspirin in 22071 initially healthy male US physicians in the primary prevention of cancer and CVD. All protocols were approved by the Institutional Review Board of Brigham and Women's Hospital.
\end{abstract}

\section{Blood collection}

A follow-up blood collection was conducted among 18764 randomised men still alive starting in December 1995. Blood kits with vacuum tubes containing EDTA, instructions for the blood draws, and coldpacks were mailed to all 18764 participants. Physicians returned blood samples in

Abbreviations: CRP, C-reactive protein; HDL-C, HDL-cholesterol; ICAM-1, intercellular adhesion molecule-1; LDL-C, LDL-cholesterol; PHS, Physicians' Health Study.

* Corresponding author: Wildon R. Farwell, fax +1 857364 4424, email Wildon.Farwell@va.gov 
provided coldpacks by prepaid overnight courier. Upon receipt, each sample was centrifuged, divided into samples, and stored at $-82^{\circ} \mathrm{C}$. Follow-up blood kits were received from $11718(62 \%)$ of 18764 participants alive at the time of the second blood collection beginning in 1995. A total of 492 middle-aged and older men who contributed blood and were identified as free of CVD and cancer were selected for a nested case-control study of plasma carotenoids and $\mathrm{CVD}^{(10)}$. We performed a cross-sectional analysis on these men identified as controls for this previous study.

Blood samples were analysed for lycopene, $\beta$-cryptoxanthin, $\alpha$-carotene, $\beta$-carotene, lutein, zeaxanthin, $\alpha$-tocopherol and $\gamma$-tocopherol at Our Lady of Mercy Medical Center (Bronx, NY, USA), a laboratory that has participated in the US Quality Assurance Program. All assays were quantified by reversed-phase HPLC after extraction and concentration by conventional methods ${ }^{(11)}$. Mean intra-assay $\mathrm{CV}$ based on blinded quality-control samples ranged from $7.6 \%$ for $\alpha$-carotene to $11.9 \%$ for $\alpha$-tocopherol. Plasma lipids, including total cholesterol, HDL-cholesterol (HDL-C) and LDL-cholesterol (LDL-C) were also assayed using commercially available diagnostic kits (Sigma-Aldrich Chemical Co., St Louis, MO, USA) and conventional methods ${ }^{(12,13)}$. Levels of C-reactive protein (CRP) were assayed using a validated, high-sensitivity assay (Denka Seiken Company, Tokyo, Japan). Levels of IL-6 and intercellular adhesion molecule-1 (ICAM-1) were assayed using commercially available enzyme-linked immunoadsorbent assays (R\&D Systems; Minneapolis, MN, USA).

\section{Baseline characteristics}

In addition to returning a blood sample, participants completed a comprehensive questionnaire that included information on self-reported clinical and lifestyle characteristics. For those who did not return a comprehensive questionnaire at the same time as their blood sample, we used questionnaires that were completed closest to the time of blood collection, within a few years, to ensure the complete assessment of potential confounding for our analyses. Questionnaires provided information on age (years), weight and height (converted to BMI; $\mathrm{kg} / \mathrm{m}^{2}$ ), smoking status (categorised as current, former, or never), alcohol use (categorised as rarely or never, monthly, weekly, or daily), frequency of exercise (categorised as rarely or never, $<3 \mathrm{~d}$ per week, 3-4d per week, or $\geq 5 \mathrm{~d}$ per week), history of hypertension (yes or no), history of hyperlipidaemia (yes or no), history of diabetes mellitus (yes or no) and systolic blood pressure ( $\mathrm{mmHg}$ ).

\section{Data analysis}

Total plasma carotenoids were defined as the sum of plasma $\alpha$-carotene, $\beta$-carotene, lycopene, zeaxanthin, lutein and $\beta$-cryptoxanthin. We defined quartiles of total plasma carotenoids based upon their distribution among controls: $<0.995,0.995-1.300,1.301-1.715$ and $>1.715 \mu \mathrm{mol} / \mathrm{l}$. We first compared participants for clinical and lifestyle characteristics by quartile of total plasma carotenoids. Crude Spearman correlation tests were first calculated for total and individual carotenoids, followed by adjustment for age and total cholesterol. Linear regression models then calculated the parameter estimate and $95 \%$ CI for levels of total plasma carotenoids and each variable after adjustment for total cholesterol. Subsequent models further adjusted for three classes of variables - clinical, lifestyle and biomarkers - as well as a model that included all covariates. We also considered forward, backward and stepwise selection procedures to identify which combination of variables provided the best and most efficient fit of the data.

Next, we used logistic regression models to calculate the OR and $95 \% \mathrm{CI}$ for having total plasma carotenoids that are above $v$. below the median value $(1.301 \mu \mathrm{mol} / \mathrm{l})$. We followed a similar model-building strategy as described above. We considered full multivariate models comparing the upper quartile of total plasma carotenoids $(>1.715 \mu \mathrm{mol} / \mathrm{l})$ with the bottom quartile of total plasma carotenoids $(<0.950 \mu \mathrm{mol} / \mathrm{l})$. Finally, we considered the use of a multivitamin in our multivariate models. All statistical analyses were performed using SAS (version 9.1; Cary, NC, USA).

\section{Results}

For all 492 men, the mean age in this analysis was 69.6 (SD 8.0) years. For individual carotenoids, the mean levels of each individual carotenoid were as follows: lycopene, 0.18 (SD 0.09) $\mu \mathrm{mol} / \mathrm{l} ; \quad \beta$-cryptoxanthin, $0.20 \quad$ (SD 0.15) $\mu \mathrm{mol} / \mathrm{l}$; lutein, 0.28 (SD 0.12) $\mu \mathrm{mol} / \mathrm{l}$; zeaxanthin, 0.09 (SD $0.05) \mu \mathrm{mol} / \mathrm{l} ; \alpha$-carotene, 0.14 (SD 0.13) $\mu \mathrm{mol} / \mathrm{l} ; \beta$-carotene, $0.56(\mathrm{SD} 0.51) \mu \mathrm{mol} / \mathrm{l}$. The mean level of total plasma carotenoids for the study population was 1.45 (SD 0.71) $\mu \mathrm{mol} / \mathrm{l}$. Table 1 presents the characteristics of the population by quartiles of total plasma carotenoids. Increasing levels of total plasma carotenoids were not associated with age or BMI. Significantly fewer men had a diagnosis of hypertension, smoked or drank alcohol with increasing levels of total plasma carotenoids. Total cholesterol, LDL-C and HDL-C each significantly increased with higher quartiles of total plasma carotenoids. Finally, as would be expected, individual plasma carotenoids significantly increased with increasing levels of total plasma carotenoids.

Table 2 presents Spearman correlations among individual and total plasma carotenoids after controlling for total cholesterol and age. No significant differences were found for crude $v$. adjusted Spearman correlation coefficients. Individual plasma carotenoids were significantly correlated with one another as well as total plasma carotenoids. After zeaxanthin and lutein, the next highest Spearman correlation among individual carotenoids occurred between $\alpha$-carotene and $\beta$-carotene $(r 0.66 ; P<0 \cdot 01)$.

Forward, backward and stepwise selection methods were used to generate and compare linear regression models that may predict total plasma carotenoids. The maximum $r^{2}$ in each selection process was 0.17 with total cholesterol, LDL-C, HDL-C, BMI, daily alcohol ingestion, weekly alcohol ingestion, current smoking, diagnosis of hypertension, ICAM-1, $\alpha$-tocopherol and $\gamma$-tocopherol statistically significant in each model for total plasma carotenoids (data not shown). Table 3 presents results from the linear regression models for total plasma carotenoids. Among the clinical variables, diagnoses of diabetes mellitus and hyperlipidaemia were not significant predictors of increased total plasma carotenoids in any model, whereas a diagnosis of hypertension and 
Table 1. Characteristics of 492 middle-aged and older male physicians by quartiles of total plasma carotenoids*

(Mean values and standard deviations for 123 subjects per quartile)

\begin{tabular}{|c|c|c|c|c|c|c|c|c|c|}
\hline \multirow[t]{2}{*}{ Quartiles of total plasma carotenoids... } & \multicolumn{2}{|c|}{$1(<0.995 \mu \mathrm{mol} / \mathrm{l})$} & \multicolumn{2}{|c|}{$\begin{array}{c}2(0.995- \\
1.300 \mu \mathrm{mol} / \mathrm{l})\end{array}$} & \multicolumn{2}{|c|}{$\begin{array}{c}3(1.301- \\
1.715 \mu \mathrm{mol} / \mathrm{l})\end{array}$} & \multicolumn{2}{|c|}{$4(>1.715 \mu \mathrm{mol} / \mathrm{l})$} & \multirow[b]{2}{*}{$P$ for trend } \\
\hline & Mean & SD & Mean & SD & Mean & SD & Mean & SD & \\
\hline Subjects $(n)$ & \multicolumn{2}{|c|}{123} & \multicolumn{2}{|c|}{123} & \multicolumn{2}{|c|}{123} & \multicolumn{2}{|c|}{123} & \\
\hline Age (years) & $69 \cdot 6$ & $8 \cdot 2$ & $70 \cdot 5$ & $7 \cdot 6$ & 68.9 & $8 \cdot 7$ & $69 \cdot 5$ & $7 \cdot 6$ & 0.29 \\
\hline BMI $\left(\mathrm{kg} / \mathrm{m}^{2}\right)$ & $26 \cdot 3$ & 3.7 & $25 \cdot 2$ & 3.0 & 25.5 & $3 \cdot 0$ & $25 \cdot 7$ & $14 \cdot 3$ & 0.88 \\
\hline Systolic blood pressure (mmHg) & $131 \cdot 8$ & 13.5 & $128 \cdot 9$ & $12 \cdot 3$ & $130 \cdot 3$ & $12 \cdot 4$ & $127 \cdot 2$ & $12 \cdot 1$ & 0.03 \\
\hline Diagnosis of diabetes mellitus (\%) & \multicolumn{2}{|c|}{$5 \cdot 7$} & \multicolumn{2}{|c|}{5.0} & \multicolumn{2}{|c|}{1.6} & \multicolumn{2}{|c|}{$4 \cdot 1$} & 0.30 \\
\hline Diagnosis of hyperlipidaemia (\%) & \multicolumn{2}{|c|}{$16 \cdot 7$} & \multicolumn{2}{|c|}{$14 \cdot 3$} & \multicolumn{2}{|c|}{$19 \cdot 7$} & \multicolumn{2}{|c|}{$13 \cdot 2$} & 0.74 \\
\hline Smoking status (\%) & & & & & & & & & $<0.01$ \\
\hline Never & \multicolumn{2}{|c|}{$43 \cdot 1$} & \multicolumn{2}{|c|}{$43 \cdot 1$} & \multicolumn{2}{|c|}{52.9} & \multicolumn{2}{|c|}{55.3} & \\
\hline Former & & & & & & & & & \\
\hline Current & & & & & & & & & \\
\hline Exercise (\%) & & & & & & & & & 0.14 \\
\hline Rarely or never & & & & & & & & & \\
\hline$\geq 5 \mathrm{~d} /$ week & & & & & & & & & \\
\hline Alcohol consumption (\%) & & & & & & & & & $<0.01$ \\
\hline Rarely/never & & & & & & & & & \\
\hline Monthly & & & & & & & & & \\
\hline Weekly & & & & & & & & & \\
\hline Daily & & & & & & & & & \\
\hline Plasma total cholesterol (mg/l) & 2043 & 413 & 2179 & 429 & 2227 & 398 & 2199 & 469 & $<0.01$ \\
\hline Plasma LDL-C (mg/l) & 1312 & 332 & 1428 & 367 & 1471 & 367 & 1452 & 401 & $<0.01$ \\
\hline Plasma HDL-C (mg/l) & 426 & 121 & 481 & 157 & 490 & 135 & 491 & 131 & $<0.01$ \\
\hline Plasma CRP (mg/l) & $3 \cdot 2$ & 4.9 & 2.9 & 3.7 & $2 \cdot 8$ & 4.3 & $2 \cdot 0$ & 2.7 & 0.05 \\
\hline Plasma ICAM-1 (ng/ml) & $324 \cdot 2$ & 123.5 & $299 \cdot 8$ & $89 \cdot 3$ & 291.3 & $67 \cdot 1$ & 281.5 & $59 \cdot 2$ & $<0.01$ \\
\hline Plasma IL-6 (pg/ml) & 2.27 & 1.58 & 2.55 & 3.51 & 1.88 & 1.45 & $2 \cdot 24$ & $3 \cdot 21$ & 0.68 \\
\hline$\alpha$-Tocopherol $(\mu \mathrm{mol} / \mathrm{l})$ & $34 \cdot 6$ & $18 \cdot 4$ & $35 \cdot 8$ & 14.9 & $36 \cdot 7$ & $14 \cdot 2$ & 39.5 & $15 \cdot 9$ & 0.03 \\
\hline$\gamma$-Tocopherol $(\mu \mathrm{mol} / \mathrm{l})$ & $4 \cdot 27$ & 3.02 & 4.48 & $3 \cdot 11$ & 4.09 & 2.39 & $4 \cdot 23$ & $3 \cdot 24$ & 0.51 \\
\hline Plasma lycopene $(\mu \mathrm{mol} / \mathrm{l})$ & 0.13 & 0.06 & 0.19 & 0.09 & 0.20 & 0.07 & 0.22 & 0.10 & $<0.01$ \\
\hline Plasma $\beta$-cryptoxanthin ( $\mu \mathrm{mol} / \mathrm{l})$ & $0 \cdot 10$ & 0.06 & 0.17 & $0 \cdot 10$ & 0.23 & 0.13 & 0.31 & 0.19 & $<0.01$ \\
\hline Plasma lutein $(\mu \mathrm{mol} / \mathrm{l})$ & $0 \cdot 19$ & 0.06 & 0.27 & 0.08 & 0.30 & $0 \cdot 10$ & 0.36 & 0.16 & $<0.01$ \\
\hline Plasma $\beta$-carotene $(\mu \mathrm{mol} / \mathrm{l})$ & 0.22 & $0 \cdot 11$ & 0.35 & 0.13 & 0.52 & 0.17 & $1 \cdot 13$ & 0.70 & $<0.01$ \\
\hline
\end{tabular}

LDL-C, LDL-cholesterol; HDL-C, HDL-cholesterol; CRP, C-reactive protein; ICAM-1, intercellular adhesion molecule-1.

${ }^{*}$ For details of procedures, see Methods.

BMI were significant predictors in all three models. Among the lifestyle variables, daily and weekly alcohol intakes were significant predictors of increased total plasma carotenoids in all three models. Among the biomarkers, ICAM-1, HDL-C and $\alpha$-tocopherol were significant predictors of increased total plasma carotenoids in all three models.
Table 4 presents results from total cholesterol-adjusted, class-adjusted and multivariate-adjusted logistic regression models comparing those above $v$. below the median level of total plasma carotenoids. Among clinical variables, no variable was associated with increased levels of total plasma carotenoids in the overall multivariate-adjusted model.

Table 2. Spearman correlations for plasma levels of each individual carotenoid and total carotenoids controlled for total cholesterol and age among middle-aged and older male physicians*

\begin{tabular}{|c|c|c|c|c|c|c|c|c|c|c|c|c|}
\hline & \multicolumn{2}{|c|}{$\begin{array}{c}\beta \text {-Crypto- } \\
\text { xanthin }\end{array}$} & \multicolumn{2}{|c|}{ Lycopene } & \multicolumn{2}{|c|}{$\alpha$-Carotene } & \multicolumn{2}{|c|}{$\beta$-Carotene } & \multicolumn{2}{|c|}{ Lutein } & \multicolumn{2}{|c|}{ Zeaxanthin } \\
\hline & $r$ & $P$ & $r$ & $P$ & $r$ & $P$ & $r$ & $P$ & $r$ & $P$ & $r$ & $P$ \\
\hline Lycopene & 0.16 & $<0.01$ & \multicolumn{2}{|r|}{-} & \multicolumn{2}{|c|}{-} & \multicolumn{2}{|c|}{-} & \multicolumn{2}{|c|}{-} & \multicolumn{2}{|c|}{ - } \\
\hline$\alpha$-Carotene & 0.27 & $<0.01$ & 0.25 & $<0.01$ & \multicolumn{2}{|c|}{-} & \multicolumn{2}{|c|}{-} & \multicolumn{2}{|c|}{-} & \multicolumn{2}{|c|}{-} \\
\hline$\beta$-Carotene & 0.35 & $<0.01$ & 0.24 & $<0.01$ & 0.66 & $<0.01$ & \multicolumn{2}{|c|}{-} & \multirow{2}{*}{\multicolumn{2}{|c|}{-}} & \multicolumn{2}{|c|}{-} \\
\hline Lutein & 0.37 & $<0.01$ & 0.22 & $<0.01$ & 0.28 & $<0.01$ & 0.17 & $<0.01$ & & & \multicolumn{2}{|c|}{-} \\
\hline Zeaxanthin & 0.36 & $<0.01$ & 0.22 & $<0.01$ & 0.27 & $<0.01$ & 0.17 & $<0.01$ & $>0.99$ & $<0.01$ & \multicolumn{2}{|c|}{-} \\
\hline Total plasma carotenoids & 0.58 & $<0.01$ & 0.38 & $<0.01$ & 0.71 & $<0.01$ & 0.86 & $<0.01$ & 0.49 & $<0.01$ & 0.49 & $<0.01$ \\
\hline
\end{tabular}

${ }^{*}$ For details of procedures and subjects, see Methods and Table 1. 
Table 3. Parameter estimates for total plasma carotenoids from total cholesterol-adjusted, class-adjusted and overall multivariate-adjusted linear regression models ${ }^{*}$ (Parameter estimates and $95 \%$ confidence intervals)

\begin{tabular}{|c|c|c|c|c|c|c|}
\hline & \multicolumn{6}{|c|}{ Parameter estimate } \\
\hline & Total cholesterol-adjusted & $95 \% \mathrm{Cl}$ & Class-adjusted $\dagger$ & $95 \% \mathrm{Cl}$ & Overall multivariate-adjusted $\ddagger$ & $95 \% \mathrm{Cl}$ \\
\hline \multicolumn{7}{|l|}{ Clinical variables } \\
\hline Age (per 10 years) & -0.01 & $-0.09,0.07$ & 0.01 & $-0.07,0.10$ & 0.03 & $-0.06,0.12$ \\
\hline BMI $\left(\right.$ per $\left.5 \mathrm{~kg} / \mathrm{m}^{2}\right)$ & 0.05 & $0.01,0.09$ & 0.07 & $0.02,0.11$ & 0.08 & $0.04,0.12$ \\
\hline Systolic blood pressure (per $10 \mathrm{mmHg}$ ) & -0.06 & $-0.11,-0.01$ & -0.04 & $-0.10,0.02$ & -0.02 & $-0.08,0.04$ \\
\hline Diagnosis of hypertension & -0.22 & $-0.35,-0.10$ & -0.20 & $-0.35,-0.05$ & -0.20 & $-0.35,-0.06$ \\
\hline Diagnosis of diabetes mellitus & -0.03 & $-0.34,0.29$ & -0.07 & $-0.41,0.28$ & -0.04 & $-0.38,0.30$ \\
\hline Diagnosis of hyperlipidaemia & -0.07 & $-0.24,0.10$ & -0.08 & $-0.25,0.10$ & -0.15 & $-0.33,0.03$ \\
\hline \multicolumn{7}{|l|}{ Lifestyle variables } \\
\hline \multicolumn{7}{|l|}{ Smoking } \\
\hline Never smoked & \multicolumn{2}{|c|}{ Reference } & \multicolumn{2}{|c|}{ Reference } & \multicolumn{2}{|l|}{ Reference } \\
\hline Quit smoking & -0.09 & $-0.22,0.04$ & -0.07 & $-0.19,0.06$ & -0.11 & $-0.24,0.03$ \\
\hline Current smoking & -0.52 & $-0.79,-0.24$ & -0.44 & $-0.73,-0.16$ & -0.28 & $-0.59,0.02$ \\
\hline \multicolumn{7}{|l|}{ Exercise } \\
\hline Rarely or never exercise & \multicolumn{2}{|c|}{ Reference } & \multicolumn{2}{|c|}{ Reference } & \multicolumn{2}{|l|}{ Reference } \\
\hline Exercise $<3 \mathrm{~d}$ per week & 0.07 & $-0.11,0.24$ & 0.05 & $-0.13,0.22$ & 0.06 & $-0.12,0.24$ \\
\hline Exercise $3-4 d$ per week & 0.07 & $-0.09,0.23$ & 0.03 & $-0.13,0.19$ & 0.08 & $-0.09,0.24$ \\
\hline Exercise $\geq 5 \mathrm{~d}$ per week & 0.11 & $-0.07,0.29$ & 0.06 & $-0.12,0.24$ & 0.08 & $-0.11,0.27$ \\
\hline \multicolumn{7}{|l|}{ Alcohol intake } \\
\hline Rare or never alcohol ingestion & \multicolumn{2}{|c|}{ Reference } & \multicolumn{2}{|c|}{ Reference } & \multicolumn{2}{|l|}{ Reference } \\
\hline Monthly alcohol ingestion & 0.09 & $-0.11,0.29$ & 0.06 & $-0.14,0.26$ & 0.11 & $-0.10,0.33$ \\
\hline Weekly alcohol ingestion & 0.28 & $0.06,0.51$ & 0.25 & $0.03,0.48$ & 0.36 & $0.12,0.60$ \\
\hline Daily alcohol ingestion & 0.26 & $0.08,0.44$ & 0.21 & $0.03,0.40$ & 0.28 & $0.08,0.47$ \\
\hline \multicolumn{7}{|l|}{ Biomarkers } \\
\hline Total cholesterol (per $100 \mathrm{mg} / \mathrm{l}$ ) & 0.02 & $0.00,0.03$ & -0.09 & $-0.15,-0.03$ & -0.09 & $-0.16,-0.03$ \\
\hline LDL-C (per $100 \mathrm{mg} / \mathrm{l})$ & 0.01 & $-0.04,0.06$ & 0.11 & $0.04,0.17$ & 0.11 & $0.04,0.18$ \\
\hline HDL-C (per $100 \mathrm{mg} / \mathrm{l})$ & 0.06 & $0.01,0.11$ & 0.12 & $0.06,0.18$ & 0.14 & $0.08,0.21$ \\
\hline CRP (per $10 \mathrm{mg} / \mathrm{l})$ & -0.20 & $-0.36,-0.04$ & -0.15 & $-0.31,0.02$ & -0.10 & $-0.26,0.07$ \\
\hline ICAM-1 (per $100 \mathrm{ng} / \mathrm{ml})$ & -0.10 & $-0.17,-0.03$ & -0.08 & $-0.15,-0.01$ & -0.08 & $-0.15,0.00$ \\
\hline IL-6 (per $100 \mathrm{pg} / \mathrm{ml})$ & -1.29 & $-3 \cdot 68,1 \cdot 11$ & -0.76 & $-3.18,1.66$ & -0.85 & $-3.34,1.64$ \\
\hline$\alpha$-Tocopherol $($ per $10 \mu \mathrm{mol} / \mathrm{l})$ & 0.04 & $0.00,0.08$ & 0.09 & $0.04,0.14$ & 0.10 & $0.05,0.15$ \\
\hline$\gamma$-Tocopherol $($ per $1 \mu \mathrm{mol} / \mathrm{l})$ & -0.01 & $-0.03,0.01$ & 0.02 & $-0.01,0.04$ & 0.03 & $0.00,0.05$ \\
\hline
\end{tabular}

LDL-C, LDL-cholesterol; HDL-C, HDL-cholesterol; CRP, C-reactive protein; ICAM-1, intercellular adhesion molecule-1.

For details of procedures and subjects, see Methods and Table 1.

†Each multivariate model controlled for total cholesterol and each variable listed within each variable class.

‡Each multivariate model controlled for total cholesterol and every other variable listed. 
Table 4. Odds ratios for above $v$. below the median value $(1.301 \mu \mathrm{mol} / \mathrm{l})$ of total plasma carotenoids from total cholesterol-adjusted, class-adjusted and overall multivariate-adjusted logistic regression models*

(Odds ratios and $95 \%$ confidence intervals)

OR

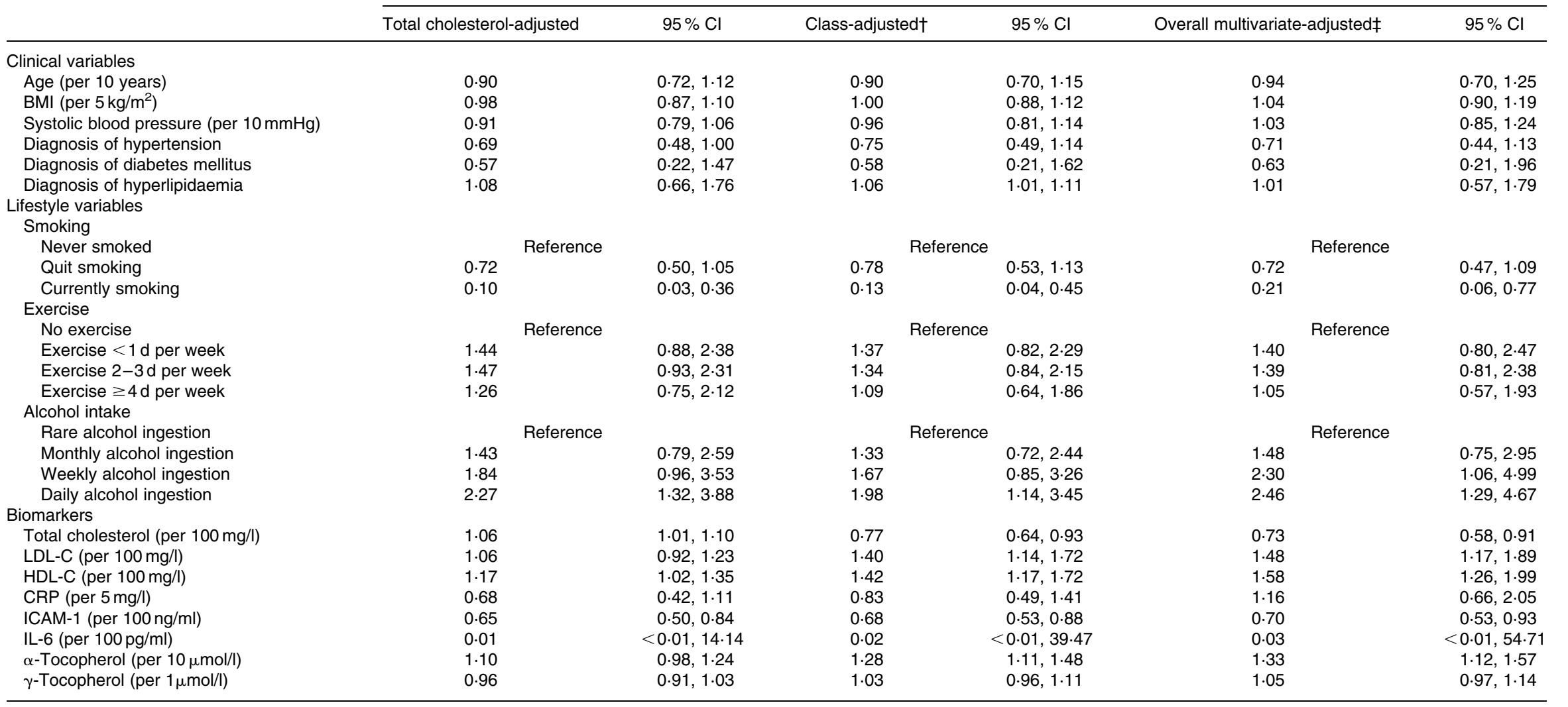

LDL-C, LDL-cholesterol; HDL-C, HDL-cholesterol; CRP, C-reactive protein; ICAM-1, intercellular adhesion molecule-1.

For details of procedures and subjects, see Methods and Table 1.

†Each multivariate model controlled for total cholesterol and each variable listed within each variable class.

$\ddagger$ Each multivariate model controlled for total cholesterol and every other variable listed. 
Among the lifestyle variables, the OR and $95 \%$ CI were statistically significant in all three models for current smoking (OR 0.10 (95\% CI $0.03,0.36$ ), OR 0.13 (95\% CI 0.04 , 0.45 ) and OR 0.21 (95\% CI $0.06,0.77)$, respectively) and daily alcohol ingestion (OR 2.27 (95\% CI 1.32, 3.88), OR 1.98 (95\% CI $1.14,3.45)$ and OR 2.46 (95\% CI 1.29, 4.67), respectively). No other lifestyle variables had a significant association in any model. Significant positive associations with total plasma carotenoids were found for each $100 \mathrm{mg} / \mathrm{l}$ difference in LDL-C and $10 \mu \mathrm{mol} / \mathrm{l}$ difference in $\alpha$-tocopherol in the class-adjusted and overall multivariate-adjusted models only. However, the OR for each $100 \mathrm{mg} / \mathrm{l}$ difference in HDL-C was statistically significant in all three models (OR $1.17(95 \%$ CI 1.02, 1.35), OR 1.42 (95\% CI 1.17, 1.72) and OR 1.58 ( $95 \%$ CI 1.26, 1.99), respectively). Including the use of a multivitamin in the overall multivariate model did not significantly alter these results (data not shown).

We also compared men in the highest quartile of total plasma carotenoids with men in the lowest quartile using multivariateadjusted logistic regression models. The only statistically significant OR were for $10 \mathrm{mmHg}$ difference in systolic blood pressure (OR 0.66; $95 \%$ CI 0.47, 0.93), daily alcohol ingestion (OR 11.38; $95 \%$ CI 3.37, 38.45), weekly alcohol ingestion (OR $9.45 ; 95 \%$ CI 2.45, 36.43), monthly alcohol ingestion (OR $4.57 ; 95 \%$ CI $1.28,16.28), 100 \mathrm{mg} / \mathrm{l}$ increase in total cholesterol (OR $0.54 ; 95 \%$ CI $0.36,0.80), 100 \mathrm{mg} / 1$ increase in LDL-C (OR 2.21; $95 \%$ CI 1.43, 3.42), $100 \mathrm{mg} / \mathrm{l}$ increase in HDL-C (OR 2.41; $95 \%$ CI 1.62, 3.61), $100 \mathrm{ng} / \mathrm{ml}$ increase in ICAM-1 (OR $0.53 ; 95 \% \mathrm{CI} 0.29,0.95)$ and $10 \mu \mathrm{mol} / \mathrm{l}$ increase in $\alpha$-tocopherol (OR 1.83; $95 \%$ CI 1.39, 2.41). Including the use of a multivitamin in the overall multivariate model did not significantly alter these results (data not shown).

\section{Discussion}

In the present cross-sectional study examining potential determinants of total plasma carotenoids in middle-aged and older men, few lifestyle and clinical risk factors appear to be related to levels of total plasma carotenoids. Only current smoking and daily alcohol ingestion appeared to be significantly associated with total plasma carotenoid levels. On the other hand, plasma lipids and $\alpha$-tocopherol were strongly associated with total plasma carotenoid levels. More novel inflammatory biomarkers such as CRP, ICAM-1 and IL-6 were not associated with total plasma carotenoids when considered simultaneously with lipids and tocopherols. Of the individual carotenoids, levels of $\beta$-carotene and $\alpha$-carotene correlated the most with levels of total plasma carotenoids.

The noted inverse correlation between smoking and levels of total plasma carotenoid has been observed in other studies ${ }^{(14,15)}$. Data from the ' $\beta$-Carotene and Retinol Efficacy Trial' (CARET) showed that for every ten cigarettes smoked per $d$, serum $\beta$-carotene concentration was $5.4 \%$ lower $^{(16)}$. Proposed mechanisms for the inverse relationship between total plasma carotenoid levels and cigarette smoking include decreased absorption of dietary carotenoids among active smokers and increased clearance of plasma carotenoids as a consequence of their interaction with free radicals produced as a result of cigarette smoking ${ }^{(17)}$.
Dietary characteristics may partially explain the observed associations among HDL-C, $\alpha$-tocopherol and total plasma carotenoids. Studies have shown that diets high in unsaturated fat may increase the absorption of individual and total carotenoids ${ }^{(18-20)}$. Dietary sources of unsaturated fats also tend to be sources of tocopherols. In addition, diets high in unsaturated fats are associated with increased levels of plasma HDL$\mathrm{C}^{(21)}$. Another possible explanation for the relationship between carotenoids and tocopherols is that physicians may be more inclined to take multivitamin supplements that include both carotenoids and tocopherols. Unfortunately, we did not have information on the components of individual diets or multivitamin supplements for this analysis.

Previous studies have shown an inverse correlation between inflammatory markers and levels of carotenoids ${ }^{(19,20,22,23)}$. The Mediterranean diet may also be associated with increased serum levels of carotenoids and decreased serum levels of inflammatory markers such as $\operatorname{CRP}^{(19,20)}$. One study found a statistically significant inverse correlation between serum levels of ICAM-1 and both lutein and lycopene as well as between CRP and $\beta$-carotene ${ }^{(23)}$. In our analysis, serum levels of each inflammatory marker decreased with increasing levels of total plasma carotenoids. However, in multivariate analyses, we did not find CRP, IL-6 or ICAM-1 to be statistically significant predictors for levels of total plasma carotenoids when we simultaneously considered other biomarkers such as lipids and tocopherols.

The present cross-sectional study examines plasma carotenoids with a comprehensive assortment of variables, but some potentially important limitations remain. First, this analysis is cross-sectional from which no causal relationships can be directly assessed. Second, we did not have specific information on diet or supplements. Third, we did not have information regarding the number of cigarettes consumed or the amount of alcohol consumed by these men. Though we relied upon selfreports from our physician participants, healthcare providers have been shown to be accurate reporters of their health status $^{(24)}$. Finally, our analyses were limited to predominantly white, apparently healthy, middle-aged and older male physicians and therefore may not apply to women, men with poor health, younger men, or men at other socioeconomic levels. There may be differences in plasma carotenoids according to sex, ethnic and cultural groups ${ }^{(4-7)}$ that warrant additional research.

In conclusion, total plasma carotenoid levels were primarily influenced by smoking, alcohol ingestion, lipid parameters and $\alpha$-tocopherol. We found that current smoking was associated with lower levels of total plasma carotenoids, while daily alcohol ingestion, increasing plasma levels of LDL-C, HDL-C and $\alpha$-tocopherol were each significantly associated with increasing levels of total plasma carotenoids. Understanding the predictors of total plasma carotenoids may improve our understanding of risk factors for chronic conditions such as CVD and cancer.

\section{Acknowledgements}

The present study was funded by NIH CA 97193 and BASF AG, plus a grant from Roche Vitamins, Inc. Approximately half of the participants in PHS II also participated in PHS I, 
which was established through NIH CA 34944, CA 40360, HL 26490 and HL 34595 . Work on the present study was also supported by the Cooperative Studies Program of the Department of Veterans Affairs Office of Research and Development.

This research was supported in part by a grant from Roche Vitamins, Inc. The authors have no other financial or personal interests related to this research. The authors would like to acknowledge the crucial contributions of the entire staff of the PHS. We are also indebted to the 29071 dedicated and committed participants randomised into the PHS starting in either 1982 or 1995.

\section{References}

1. Fairfield KM \& Fletcher RH (2002) Vitamins for chronic disease prevention in adults. JAMA 287, 3116-3126.

2. Mares-Perlman JA, Millen AE, Ficek TL \& Hankinson SE (2002) The body of evidence to support a protective role for lutein and zeaxanthin in delaying chronic disease. Overview. J Nutr 132, 518S-524S.

3. Voutilainen S, Nurmi T, Mursu J \& Rissanen TH (2006) Carotenoids and cardiovascular health. Am J Clin Nutr 83, 1265-1271.

4. Brady WE, Mares-Perlman JA, Bowen P \& Stacewicz-Sapuntzakis M (1996) Human serum carotenoid concentrations are related to physiologic and lifestyle factors. J Nutr 126, 129-137.

5. Ganji V \& Karai MR (2005) Population determinants of serum lycopene concentrations in the United States: data from the Third National Health and Nutrition Examination Survey, 1988-1994. J Nutr 135, 567-572.

6. Neuhouser ML, Rock CL, Eldrige AL, Kristal AR, Patterson RE, Cooper DA, Neumark-Sztainer D, Cheskin LJ \& Thornquist MD (2001) Serum concentrations of retinol, $\alpha$-tocopherol and the carotenoids are influenced by diet, race and obesity in a sample of healthy adolescents. J Nutr 131, 2184-2191.

7. Rock CL, Thornquist MD, Neuhouser ML, Kristal AR, Neumark-Sztainer D, Cooper DA, Patterson RE \& Cheskin LJ (2002) Diet and lifestyle correlates of lutein in the blood and diet. Symposium: can lutein protect against chronic disease? J Nutr 132, 525S-530S.

8. Le Marchand L, Hankin JH, Carter FS, Essling C, Luffey D, Franke AA, Wilkens LR, Cooney RV \& Kolonel LN (1994) A pilot study on the use of plasma carotenoids and ascorbic acid as markers of compliance to a high fruit and vegetable dietary intervention. Cancer Epidemiol Biomarkers Prev 3, 245-251.

9. Hennekens CH, Buring JE, Manson JE, et al. (1996) Lack of effect of long-term supplementation with $\beta$ carotene on the incidence of malignant neoplasms and cardiovascular disease. $N$ Engl J Med 334, 1145-1149.

10. Sesso HD, Buring JE, Norkus EP \& Gaziano JM (2005) Plasma lycopene, other carotenoids, and retinol and the risk of cardiovascular disease in men. Am J Clin Nutr 81, 990-997.

11. Sowell AL, Huff DL, Yeager PR, Caudill SP \& Gunter EW (1994) Retinol, $\alpha$-tocopherol, lutein/zeaxanthin, $\beta$-cryptoxanthin, lycopene, $\alpha$-carotene, trans- $\beta$-carotene, and four retinyl esters in serum determined simultaneously by reversed-phase HPLC with multiwave length detection. Clin Chem 40 411-416.

12. Allain CC, Poon LS, Chan CS, Richmond W \& Fu PC (1974) Enzymatic determination of total serum cholesterol. Clin Chem 20, 470-475.

13. Roeschlau P, Berut E \& Gruber W (1974) Enzymatic determination of total choleserol in serum. Z Klin Chem Klin Biochem 12, 226.

14. Wallström P, Wirfält E, Lahmann PH, Gullberg B, Janzon L \& Berglund G (2001) Serum concentrations of $\beta$-carotene and $\alpha$-tocopherol are associated with diet, smoking, and general and central adiposity. Am J Clin Nutr 73, 777-785.

15. Wei W, Kim Y \& Boudreau N (2001) Association of smoking with serum and dietary levels of antioxidants in adults: NHANES III, 1988-1994. Am J Public Health 91, 258-264.

16. Goodman GE, Thornquist M, Kestlin M, Metch B, Anderson G \& Omenn GS (1996) The association between participant characteristics and serum concentrations of $\beta$-carotene, retinol, retinyl palmitate, and $\alpha$-tocopherol among participants in the carotene and retinol efficacy trial (CARET) for prevention of lung cancer. Cancer Epidemiol Biomarkers Prev 5, 815-821.

17. Northrop-Clewes CA \& Thurnham DI (2006) Monitoring micronutrients in cigarette smokers. Clinica Chimica Acta 377, 14-38.

18. Ahuja KD, Pittaway JK \& Ball MJ (2006) Effects of olive oil and tomato lycopene combination on serum lycopene, lipid profile, and lipid oxidation. Nutrition 22, 259-265.

19. Blum S, Aviram M, Ben-Amotz A \& Levy Y (2006) Effect of a Mediterranean meal on postprandial carotenoids, paraoxonase activity and C-reactive protein levels. Ann Nutr Metab 50, $20-24$.

20. Lopez-Garcia E, Schulze MB, Fung TT, Meigs JB, Rifai $\mathrm{N}$, Manson JE \& Hu FB (2004) Major dietary patterns are related to plasma concentrations of markers of inflammation and endothelial dysfunction. Am J Clin Nutr 80, $1029-1035$.

21. Sacks FM \& Katan M (2002) Randomized clinical trials on the effects of dietary fat and carbohydrate on plasma lipoproteins and cardiovascular disease. Am J Med 113, Suppl. 9B, $13 \mathrm{~S}-24 \mathrm{~S}$.

22. Gruber M, Chappell R, Millen A, LaRowe T, Moeller SM, Iannaccone A, Kritchevsky SB \& Mares J (2004) Correlates of serum lutein + zeaxanthin: findings from the Third National Health and Nutrition Examination Survey. J Nutr 134, $2387-2394$.

23. van Herpen-Broekmans WM, Klopping-Ketelaars IAA, Bots ML, Kluft C, Princen H, Hendriks HF, Tijburg LB, van Poppel G \& Kardinaal AF (2004) Serum carotenoids and vitamins in relation to markers of endothelial function and inflammation. Eur J Epidemiol 19, 915-992.

24. Colditz GA, Martin P, Stampfer MJ, Willett WC, Sampson L, Rosner B, Hennekens CH \& Speizer FE (1986) Validation of questionnaire information on risk factors and disease outcomes in a prospective cohort study of women. Am J Epidemiol $\mathbf{1 2 3}$ 894-900. 